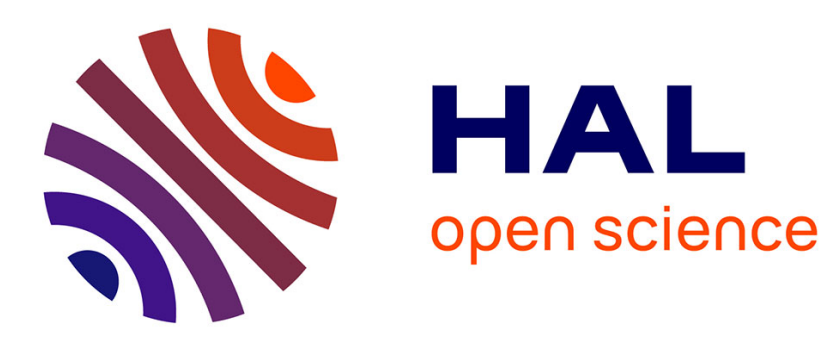

\title{
Le territoire imaginaire du "Pays Cathare". Nouvelles dynamiques du développement local en milieu rural. William Genieys
}

\section{To cite this version:}

William Genieys. Le territoire imaginaire du "Pays Cathare". Nouvelles dynamiques du développement local en milieu rural.. Pôle Sud - Revue de science politique de l'Europe méridionale, 1997, Elites, politiques et territoires, 7, pp.118-131. 10.3406/pole.1997.984 . hal-01393494

\section{HAL Id: hal-01393494 \\ https://hal.science/hal-01393494}

Submitted on 7 Nov 2016

HAL is a multi-disciplinary open access archive for the deposit and dissemination of scientific research documents, whether they are published or not. The documents may come from teaching and research institutions in France or abroad, or from public or private research centers.
L'archive ouverte pluridisciplinaire HAL, est destinée au dépôt et à la diffusion de documents scientifiques de niveau recherche, publiés ou non, émanant des établissements d'enseignement et de recherche français ou étrangers, des laboratoires publics ou privés. 


\title{
Le territoire imaginaire du "Pays Cathare"*
}

\section{Nouvelles dynamiques du développement local en milieu rural}

\author{
par William Genieys \\ CEPEL, Uninersiti Montpellier I
}

Pôle Sud $N^{\circ} 7$ - Novembre $1997-p .118$ à 131.

Le projet de développement "Pays Cathare" montre l'émergence de nouvelles formes de mobilisation du territoire. Cette étude montre l'importance du moment où les experts de l'action publique locale donnent une dimension identitaire au projet. Le choix d'une definition du "Pays Cathare" comme le territoire d'une histoire, d'un imaginaire, s'impose progressivement contre les conceptions centralistes des agents de l'Etat. Par ailleurs, cette forme de réinvention du territoire n'est pas sans effets sur la capacité politique des élites locales. Ainsi, la question de l'émergence d'un leadership territorial permet d'appréhender de façon dynamique les nouvelles articulations entre policy et politie.

\begin{abstract}
"Pays de résistance, ces contreforts du Massif central entretiennent le souvenir pas si lointain des maquis, mais aussi s'active depuis une dizaine d'années à ponctuer les parcours touristiques du "pays cathare". Cette revanche posthume des persécurés du XIII ' siècle s'appuie sur les grands sites, qui, outre le tellement fameux Montségur (dans l'Ariège), ont nom Peyrepertuse et Quéribus dans les Corbières, mais aussi Villerouge, Arques, Termes, Lastours... Aider le public dans sa découverte, sans nuire à la pureté sauvage des paysages et des panoramas, telle est la politique du département de l'Aude à laquelle un financement de l'Union européenne (un tiers dans le cadre de Lastours) apporte un renfort, au nom de la reconversion des régions économiquement défavorisées". Le Monde du 10 et 11/11/1996.
\end{abstract}

Depuis, la crise économique des années 1970 , les modes d'intervention et de gestion du territoire par l'Etat français ont changé. Les élites dirigeantes ont ainsi admis l'idée selon laquelle il fallait inventer des formes de développement adaptées à chaque situation (Thœenig, 1989, p. 116). La sociologie des politiques publiques constate à ce propos que l'étude des politiques locales est toujours en retard en France, contrastant avec la situation américaine où les recherches, les publications et les controverses se multiplient (Muller, 1990 , p. 104). Partant de là, certains sociologues ont ouvert un débat sur "les acteurs du développement local" (Sociologie $d u$
Travail, 1991). Pour ces chercheurs, il s'agissait de clarifier la réflexion sur le devenir du développement en milieu urbain au regard des lois de décentralisation (BenoitGuilbot, 1991, p. 453). Dans cette nouvelle configuration institutionnelle du pouvoir local, la dynamique des politiques de développement repose sur le jeu de quatre facteurs : une relative autonomie de décision et des ressources propres; une composition sociale favorablc de sa population (participation et consensus sur une politique locale); une position dominante dans l'espace environnant permettant l'accès aux circuits politiques pour les négociations des aides de l'État; et, enfin, une situation 


\section{Le territoire imaginaire du Pays Cathare}

historique d'opposition au pouvoir central. L'analyse de la transformation du système de gestion des villes permet de montrer que la modernisation des pratiques et des modes d'action de l'administration républicaine entraîne une transformation des rapports à l'État central (Lorrain, 1991). Le système d'administration locale tel que l'avait décrit Pierre Grémion est ainsi dépassé par le modèle du "gouvernement urbain". De façon plus générale, la politique de décentralisation était vue comme un réquilibrage volontariste entre un État trop puissant et une France profonde marginalisée au profit de l'initiative de la base (Faure, 1994). Le récent bilan des études de l'action publique des élus locaux confirme la profonde transformation des mécanismes d'allégeance des acteurs de la vie politique locale aux services administratifs déconcentrés (Duran, Thœnig, 1996). Les réformes de décentralisation contribuent au développement des pratiques de contractualisation entraînant de nouvelles formes de mobilisation et de concertation des acteurs locaux (Pôle Sud, 1996). Cette prise de conscience des limites de l'analyse sectorielle des politiques publiques est perceptible dans certains domaines de l'intervention publique tels que la lutte contre la pauvreté, l'emploi, la sécu-' rité, l'environnement ou l'urbanisme. L'analyse comparée des politiques publiques met l'accent sur les rapports qu'entretiennent les acteurs locaux à leur territoire, en faisant de la prise en compte de cette composante spatiale une condition importante de réussite de ces politiques (Balme, ali., 1994). Face à cette nouvelle configuration de la vie politique locale, certains auteurs prennent le parti d'analyser la capacité politique des ter- ritoires (Ritaine, 1994). Il s'agit alors de montrer que certains territoires dotés d'un potentiel social, économique et culturel spécifique peuvent générer des politiques particulières.

Cependant, l'analyse de la transformations des modes de l'action publique locale ne prend pas suffisamment en compte les effets induits sur les pratiques et les ressources politiques des élus locaux. La sociologie de "la vie politique" des élus locaux se limite à la description du profil social et politique des élus locaux (Rouban, Souchon-Zahn, 1996). Dans une perspective différente, la sociologie "critique" du métier politique s'intéresse à l'analyse formelle des logiques d'adaptation des élus locaux au changement social en évitant une véritable interrogation sur les pratiques politiques des politiques publiques (Fontaine, Le Bart, 1994). Afin de prolonger les critiques adressées au modèle de Grémion par les sociologues du développement urbain, il est nécessaire de dégager les corrélations entre les formes de l'action publique territorialisée et les nouveaux modes de légitimation des élites locales. Plus précisément, ce qui nous intéresse, c'est la construction de ponts entre ces deux approches. Ainsi, l'analyse d'un processus de développement local en milieu rural fournit un terrain d'étude fructueux pour la mise en chantier d'une telle liaison.

À partir de notre étude de terrain réalisée en milieu rural, le projet "Pays Cathare", on donnera une nouvel éclairage à la problématique de l'action publique locale. Cette enquête a été effectuée sur la base d'un recueil systématique des articles de presse et d'une vingtaine d'entretiens réalisés auprès des acteurs qui ont porté le projet "Pays 


\section{Pôle Sud $N^{\circ} 7$}

Cathare" entre 1983 et $1995^{\circ}$. En s'appuyant sur ces nombreux matériaux, il s'agit d'analyser ce processus de développement local dans ses différentes dimensions. On soulignera la nature particulière du projet et les stratégies des experts qui le construisent. Partant de là, on insistera sur le fait que les élus locaux impliqués mobilisent des répertoires d'action publique différenciés pour imposer leur leadership politique sur le territoire. En effet, l'analyse de la genèse d'un projet de développement à dimension identitaire permet de montrer comment, à un moment donné, des experts locaux imposent du sens sur le territoire du Pays Cathare. Alors que certains sociologues annoncent "la fin des territoires" (Badie, 1995) ou "l'illusion identitaire" (Bayart, 1996), la question de la mobilisation des identités territoriales dans la construction d'un projet de développement local sera au centre de notre analyse. Par la suite, il s'agit de dépasser notre réflexion sur la "dimension communautaire" du développement par la prise en compte de la capacité de certains élus locaux à imposer leur leadership politique. L'analyse de la mobilisation des différents répertoires de l'action publique locale par ces acteurs (logiques sectorielles versus logique territoriale) ouvre la voie à une réflexion plus générale sur les nouvelles formes de légitimation de l'action politique locale.

\section{L'institutionnalisation du territoire : nouvel enjeu du développement local?}

Le territoire du "Pays Cathare" se situe dans le sud de la France, et s'étend au pied des contreforts pyrénéens, sur deux régions et plusieurs départements ${ }^{2}$. Le département rural de l'Aude doté d'un fort patrimoine historique et culturel, les châteaux cathares, élabore dans les années 80 le programme de développement local étudié. C'est dans le contexte du processus de décentralisation institutionnelle et politique que certains acteurs locaux optent pour une stratégie de mobilisation du patrimoine culturel pour valoriser leur territoire. Dès le début des années 80 , le président du Conseil général de l'Aude prolonge la politique de la "mission Racine " en cuvrant pour l'instauration d'un tourisme dans l'arrière-pays audois. En effet, la fragile économie locale a vu sa petite industrie disparaître progressivement alors que le développement se porte sur le littoral. En raison de cette situation économique désastreuse et de la désertification du monde rural, ce notable socialiste audois s'engage dans la mise en place de nouveaux instruments devant faciliter le développement local. Grâce aux opportunités offertes par les lois de décentralisation, il met sur pied des institutions para-départementales telles que le Centre National d'Études Cathares (CNEC ${ }^{4}$ ) et le Comité Départemental du Patrimoine Culturel Audois (CDPCA) pour évaluer le patrimoine culturel de l'Aude. La dynamique du "retour des terroirs" s'appuie sur une mobilisation d'un substrat social régionaliste assez fort dans cette région (Touraine, Dubet, Wieviorka, 1981). Ces acteurs du développement local vont partir en quête d'un territoire imaginaire : le "Pays Cathare". Dans cette perspective, le projet de développement est exemplaire car il montre le rôle structurant de la référence 


\section{Le territoire imaginaire du Pays Cathare}

à un territoire imaginaire sur ce type d'action publique locale. Dans ce cas particulier, le syncrétisme stratégique entre une culture locale réifiée par des intellectuels attachés au "patrimoine cathare" (CDPCA et $\mathrm{CNEC}$ ) et la politique de reconversion de l'Aude portée par un réseau d'acteurs locaux donne naissance à une forme particulière de mobilisation du territoire qui remet en cause la dimension tutélaire de l'État français.

L'invention d'un nouveau territoire pour le développement local est posée de façon explicite par les experts locaux qui participent à l'élaboration du projet "Pays Cathare". Ce constat annonce-t-il l'apparition d'un nouveau mode de mobilisation du territoire par des acteurs en quête de nouvelles réalités sociales ou est-ce le signe du retour de "l'utopie communautaire" (Lacroix, 1981)? En effet, pourquoi mobilisent-ils un référent territorial à connotation culturelle que le tradition républicaine a refoulé de son historiographie nationale? S'agit-il d'une stratégie délibérée d'invention d'une tradition au sens de Hobsbawn? Ce projet de développement local correspond-t-il à une nouvelle forme d'institutionnalisation des périphéries' ? L'analyse de la capacité d'innovation du réseau d'experts qui porte le projet permet ainsi d'interroger la dynamique des systèmes d'action concrets au sens où l'entend la sociologie des organisations (Crozier, Friedberg, 1977, pp. 247-251). Toutefois, la prise en compte de la dimension cognitive autour de laquelle se structure l'action collective "Pays Cathare" montre comment un système d'action échappe progressivement aux acteurs qui le génèrent.
Dans cette perspective, les institutions para-départementales vont être un lieu de réflexion au sein duquel le programme voit le jour. Un groupe d'experts se structure progressivement afin de poser les bases d'un projet de développement touristique fondé sur le patrimoine culturel de l'Aude. Il est nécessaire de rappeler que le CDPCA est une émanation du Comité départemental du Tourisme dirigé par le conseiller général du canton de Chalabre. Ce dernier place à la direction de cette institution un expert du patrimoine ${ }^{6}$ qui a pour mission l'évaluation du patrimoine culturel du département de l'Aude. Pour lui, les châteaux cathares constituent l'atout majeur de ce patrimoine. Dès lors, il s'engage dans une logique de valorisation du "Pays Cathare" en fédérant, autour du CDPCA, un réseau d'acteurs très hétérogène, comprenant un représentant de la Datar, le responsable du Commissariat de Massif, le directeur de la culture du département de l'Aude, un représentant de la direction départementale de l'agriculture et de nombreux techniciens du développement. Au terme d'une collaboration de plus en plus constante, ce groupe d'experts forme un réseau finalisé autour d'un projet de développement local en milieu rural. En vue de développer les rapports économie-culture, les premières actions du CDPCA, portent sur la connaissance et l'évaluation des ressources patrimoniales du département autour des châteaux cathares. Les acteurs constituant ce réseau avancent l'idée novatrice selon laquelle la dynamique du développement local réside dans le patrimoine culturel. Pour le directeur du CDPCA, "cette terre méridionale doit puiser 


\section{Pôle Sud $N^{\circ} 7$}

aujourd'hui ses racines et la force de son devenir en cultivant la personnalité du Pays Cathare ". Incontestablement, les sites médiévaux liés à la croisade contre les albigeois représentent, dès 1982 , plus de la moitié de la fréquentation des sites, monuments et musées ouverts au public. Dès 1984, les premières enquêtes de fréquentation-satisfaction et l'étude systématique des conditions d'accueil sur les sites sont lancées ${ }^{8}$. Le patrimoine vécu jusqu'alors comme une charge devient un atout majeur pour le développement touristique. Les réappropriations du bâti par les collectivités locales s'effectuent : achats, déclarations d'utilité publique ou travaux d'office réalisés par les services de l'État. Durant cette période, l'accueil du public se normalise : billetterie, tarifs, horaires d'ouverture s'harmonisent. Le comité départemental du patrimoine met sur pied la formule des forfaits "chèques-visites" (1985-1987), avec l'appui du comité régional au tourisme Languedoc-Roussillon et la caisse nationale des monuments historiques et des sites. Face à cette effervescence, les services de l'Etat et en particulier la DATAR encouragent, le Conseil général de l'Aude à lancer une vaste expertise pour mesurer l'atout que représente le patrimoine culturel cathare. La participation des experts locaux aux rencontres internationales pour la protection du patrimoine culturel d'Avignon en 1987 leur permet d'accéder à de nouveaux savoir-faire?. Par la suite, le Conseil général engage une étude de valorisation de l'ensemble des sites dans le cadre de l'Europe $^{10}$. En 1990, à l'issue de ces travaux préalables, un programme de développement interdépartemental est élaboré par le
CDPCA. Ce projet s'impose comme une action prioritaire pour le département de l'Aude. Avant d'aborder la question de l'institutionnalisation de cette forme d'action publique locale où le politique fait son retour, il convient d'insister sur le moment où s'affirme la référence à l'imaginaire territorial du "Pays Cathare".

\section{Le retour du "Pays Cathare": de la réalité locale à la dynamique communautaire}

Les experts locaux qui inventent le projet manifestent la volonté d'imposer comme cadre d'action un territoire imaginaire : le "Pays Cathare "". En effet, lors de la genèse du programme, ces acteurs manifestent leur autonomie fonctionnelle par rapport aux agents des services administratifs déconcentrés de l'État intégrés dans le réseau. Pour ces acteurs, il s'agit de mettre sur agenda un processus de développement local qui transcende les cadres territoriaux de l'action publique locale. Ainsi, la question du sens ou encore de la dimension cognitive des politiques publiques mérite d'être posée (Faure, Pollet, Warin, 1995).

L'analyse du moment où la référence au "Pays Cathare" s'affirme comme l'appellation légitime du projet est révélatrice dans la mesure où les acteurs du réseau qui s'y affrontent ont des représentations cognitives très différentes. Ainsi, dès les premières rencontres inter-régionales et interdépartementales, entre l'Aude, l'Hérault et l'Ariège, regroupant à la fois des professionnels du tourisme (CRT/CDT) et le monde scientifique (les directeurs des archives départementales et quelques chercheurs), la 


\section{Le territoire imaginaire du Pays Cathare}

construction d'une dynamique collective $s$ 'avère difficile en raison de l'opposition structurelle entre les représentants des secteurs du tourisme et de la culture ${ }^{12}$. A ce conflit entre les partisans d'un "tourisme culturel" et les tenants de la valorisation d'un "patrimoine culturel historique", s'ajoute la stratégie des agents de la Datar consistant à faire entrer ce programme dans le cadre des routes à thèmes de la Commission Supérieure des Monuments Historiques. La logique économique des uns et la logique centralisatrice des agents de l'État se trouvent confrontées à l'imaginaire du territoire du "Pays Cathare ${ }^{13}$ ". Partant de là, le directeur du CDPCA impose une solution médiane où le territoire virtuel et interdépartemental doit être le cadre d'action du projet de développement local. Cette conception s'affirme progressivement comme légitime auprès des experts et des techniciens locaux. Cependant, le référent "Pays Cathare" fait l'objet d'un débat entre les agents du réseau. Lors des réunions de travail relatives à la labélisation du projet de développement local, on retrouve l'opposition structurelle (et structurante) entre experts et intellectuels qui défendent une acception régionaliste du "Pays Cathare" et ceux qui privilégient un programme intégré à la planification de l'espace territorial français (routes et circuits touristiques à thèmes $\left.{ }^{14}\right)$. En effet, si les agents de l'État sont d'accord sur le travail consistant à identifier et à valoriser un patrimoine historique sur un territoire particulier, ils s'opposent à une lecture trop culturaliste du Pays Cathare. Au terme de nombreuses réunions informelles ${ }^{15}$, la thématique réductrice de la "route des châteaux cathares" est écartée grâce à un argument historique logique difficilement réfutable: les monuments que l'on présente sur les sites cathares sont de châteaux frontières dont la construction est postérieure au mouvement cathare. Dans le cadre de ces discussions le directeur du CDPCA fait prévaloir sa connaissance scientifique de l'inexistence de l'architecture cathare. En effet, son travail archéologique réalisé à l'École pratique démontre que le château de Montségur a été construit par des maîtres d'œuvres français après la croisade des albigeois. En effet, il n'y a pas de châteaux "cathares", les cathares n'étant pas des bâtisseurs. Il faut alors relier l'ensemble des sites au thème "Pays Cathare" car c'est tout un pays et des identités de pays qui participaient à cette histoire plutôt que les châteaux. La référence au Pays Cathare en tant que territoire imaginaire est imposée comme cadre privilégié par le projet de développement local. À partir de fin 1989 , le programme se structure autour du label "Pays Cathare". La construction d'un projet de développement local où la mobilisation de l'identité culturelle joue un rôle central participe au processus plus général d'apparition des nouvelles formes de transactions entre intérêts et appartenances territoriales (Gaudin, 1995).

En s'appuyant sur cette dynamique, le projet de développement reçoit un accueil favorable auprès des instances européennes en obtenant le label "Année européenne du tourisme" en 1991. Fort d'une reconnaissance symbolique, les porteurs du projet partent en quête d'un financement com- 


\section{Pôle Sud $N^{\circ} 7$}

munautaire par l'intermédiaire des experts des programmes européens ${ }^{16}$. Il s'agit, alors, d'intégrer le processus de développement dans la politique d'aide communautaire du programme Leader (Leader. Impliquer la population dans le développement local, 1994). Le caractère novateur de Leader réside dans l'approche méthodologique retenue ${ }^{17}$. En effet, le programme $s$ 'applique à des territoires infrarégionaux et dans une zone rurale. Dans cette perspective, les projets de développement local postulant au programme Leader doivent valoriser les ressources endogènes du territoire à partir d'un projet intégrateur fondé sur la mise en réseau des différents acteurs locaux au sein d'un groupe d'action locale (GAL). Après un travail de formalisation du programme Pays Cathare par les experts locaux des programmes européens, le projet est présenté devant la Direction Générale VI pour intégrer le Leader. À Bruxelles le Directeur de la DG VI manifeste un grand intérêt pour le projet qui s'appuie sur la volonté de réinventer un territoire. Les hauts fonctionnaires européens appréciant l'autonomie du projet par rapport au service administratif déconcentré de l'État français vont en faire un exemple à suivre. Le projet de développement porté jusqu'alors par un réseau d'action publique local prend une nouvelle dimension dans le cadre de cette politique européenne. Cependant, l'apport du financement européen entraîne une transformation des pratiques politiques locales par l'imposition d'un cahier des charges à laquelle la subvention est assujettie. Par ailleurs, la mise sur agenda du programme Pays Cathare dans sa version Leader I va avoir des effets sur la dimension et la dynamique du système d'action local. Tout d'abord l'octroi d'une aide financière européenne importante, conditionnée à un cofinancement du Conseil général de l'Aude, entraîne un processus d'institutionnalisation du Pays Cathare restreint au cadre départemental. Ensuite, l'imposition de la constitution d'un réseau formel, le groupe d'action local ayant pour mission de piloter le projet, transforme le réseau initial au profit des élites politiques locales. Le retour du politique se traduit alors par l'apparition de nouvelles formes de régulation de l'action publique territoriale. Les politiques communautaires, notamment les fonds structurels, trouvent dans l'ancrage territorial un moyen d'expression privilégié (Smith, 1996). Le territoire du local s'impose comme le lieu où l'on peut observer comment se redéfinissent concrètement les formes de l'action publique. Dans le cadre de l'Union européenne, les nouveaux enjeux de la recomposition économique et sociale passent bien souvent par un processus de territorialisation des politiques. Celui-ci devient une ressource facilitant l'adaptation à la crise des normes étatiques et des organisations politiques nationales.

\section{Vers l'émergence de nouveaux leaderships politiques territoriaux?}

Si les élites politiques locales ont dans un premier temps laissé la gestion du projet aux experts du patrimoine et du développement local, dans un second temps, lors de la mise sur agenda du programme Leader, elles en récupèrent la maîtrise. L'analyse des 


\section{Le territoire imaginaire du Pays Cathare}

trajectoires politiques des deux principaux conseillers généraux qui se sont mobilisés pour le projet est révélatrice de la formation de nouvelles formes de leaderships politiques territoriaux.

L'analyse de l'itinéraire politique du conseiller général du canton de Chalabre constitue une illustration des pratiques politiques d'un élu en milieu rural. Sa carrière politique se fonde sur un héritage politique et sur la mise en avant de certains savoir-faire professionnels ouvrant la voie au mandat électif local. En effet, né dans l'Aude rurale, il accède à la vie professionnelle en assurant la fonction de technicien agricole au sein du SIVOM de Chalabre. Dès 1975, en s'appuyant sur sa connaissance du terrain, il devient maire de Chalabre. Fort de cette position, il développe les structures de l'intercommunalité autour de sa municipalité. En 1979, faisant suite à une demande du président Conseil général de l'Aude, il se fait élire conseiller général sur le canton de Chalabre. L'intégration de ce jeune élu au sein de l'institution départementale se traduit par l'accroissement de sa capacité d'action sur son territoire électif. Il signe un premier "contrat de pays" au nom du SIVOM de Chalabre afin d'obtenir les aides financières de l'État. Par la suite, il transforme ce SIVOM en district de développement. Son apprentissage des rôles et des pratiques politiques locales se traduit par la conquête des positions de pouvoir au sein du département. En 1979, il prend la direction du Comité Départemental du Tourisme. Ainsi, il s'octroie la maîtrise progressive d'un secteur de l'action publique locale en plein essor. Profitant, du double effet de la décentralisation et de l'arrivée au gouvernement des socialistes, il impulse une nouvelle dynamique à la politique touristique audoise. L'ouverture de son réseau relationnel sur le cabinet du Ministre du Tourisme (Henri) lui permet d'être informé sur l'orientation de ce secteur d'action publique. Ses relations privilégiées avec les sommets de l'État augmentent sa capacité locale d'innovation. Dès lors, il crée les institutions locales, le CDPCA et la maison de la montagne audoise, au sein desquelles le programme Pays Cathare prend forme. Conformément à une pratique de la délégation du travail fondée sur la compétence, il délègue la réflexion sur le projet de développement à des experts locaux. Il apparaît ainsi comme un leader qui s'entoure d'une équipe de "mercenaires" (Bailey, 1972) fondant sa légitimité politique locale sur un important pouvoir relationnel. Son comportement lors de la formulation du programme Leader Pays Cathare en constitue l'exemple le plus significatif. Il mobilise un réseau d'experts européens travaillant déjà pour le Conseil général de l'Aude à qui il confie la mission de réaliser la mise en phase du projet de développement Pays Cathare avec le programme Leader. À ce stade, son faible degré d'implication directe dans le projet joue contre lui. Il agit comme un leader qui mobilise les ressources de l'action publique de façon instrumentale. De plus, son approche principalement sectorielle du développement local s'avère limitée face à la dynamique globale et territoriale du programme Pays Cathare.

L'analyse du parcours politique de l'autre conseiller général qui porte le projet 


\section{Pôle Sud $N^{\circ} 7$}

permet d'appréhender la constitution d'un leadership politique local fondé sur la mobilisation identitaire d'un territoire. La trajectoire sociale, la nature des liens et la répartition des rôles au sein de son équipe, la mobilisation des différents réseaux d'action (locaux, européens), la capacité d'alterner le rôle de technicien et celui de porteur du projet, font de cet élu un leader politique local atypique. À la base, ce conseiller général de l'Aude rurale a un profil socioprofessionnel proche de son confrère. Il manifeste son attachement au milieu rural en participant à l'activité des foyers Léo Lagrange en tant qu'animateur de projets. Dès 1982, il participe à la mise sur pied de la fête médiévale du château de Villerouge Terménes. En 1985, il constitue une association sur le canton de Mouthoumet "pour faire du développement local". Ensuite, il parfait son savoirfaire professionnel en suivant les formations dispensées par le CNFPT sur les techniques de développement. Parallèlement, son engagement sur le terrain lui permet d'avoir la reconnaissance du conseiller général du canton de Mouthoumet qui à l'âge de 78 ans, après 28 ans de mandat, lui propose d'assumer sa succession (1988). Âgé de moins de trente ans, il devient conseiller général de l'Aude pour ensuite s'imposer comme maire de Mouthoumet. Pour mener à bien sa politique de développement local, cet élu mobilise les outils institutionnels mis à la disposition des élites locales après la décentralisation. Ainsi, il obtient la création d'un district à fiscalité propre sur le canton de Mouthoumet en 1990 ("le plus petit de France"). Jusque là son parcours politique ascendant ressemble en bien des points à celui des élus locaux audois. Paradoxalement, il stigmatise dans son discours les notables audois pour leur immobilisme. Refusant d'entrer dans les réseaux politiques nationaux du parti socialiste, il déclare: "ce que je fais sur le canton m'apporte plus que ce que je fais au département". Pour lui, le développement économique et le travail politique se rejoignent dans une stratégie de mobilisation et réappropriation des identités territoriales par la population locale. Dans cette perspective, il s'impose progressivement comme l'acteur politique qui porte le projet. En effet, il intègre dès le début le réseau d'experts du directeur du CDPCA. Face aux partisans d'une approche sectorielle du projet, il se range du côté des tenants d'une interprétation territoriale et culturelle du Pays Cathare. Après s'être démarqué rapidement des experts du patrimoine qui minorent la dimension économique du projet, il choisit la stratégie de la territorialisation de l'action publique. Pour lui, "le Pays Cathare est un programme de développement global, c'est-à-dire qu'il est autant culturel, social et économique". De fait, en dénonçant une approche de l'action publique locale par le prisme des filières (agriculture, tourisme, commerce), il déclare vouloir intégrer l'approche identitaire et territoriale dans un processus de développement économique afin d'impliquer les populations vivant sur le territoire concerné. Dans cette acception de l'activité politique locale, l'élu ne doit pas se restreindre dans "un rôle de gestionnaire" mais "redonner la vie à un territoire qui crève parce qu'il a perdu tout référent". La 


\section{Le territoire imaginaire du Pays Cathare}

mobilisation de l'identité territoriale doit déboucher sur l'établissement d'une "démarche participative" vis-à-vis des populations locales. Cette façon particulière de promouvoir le développement local lui permet de s'imposer comme le porteur légitime du programme Pays Cathare. Sa nomination à la présidence du CDPCA en 1989 en est la preuve. Par la suite, il structure autour de lui une équipe de techniciens fidèles qui partagent sa conception du développement local. Le travail de ces réseaux où chacun joue pleinement son rôle s'avère efficace lors du passage au programme Leader I. Les experts européens contactés par Jacques Montagné trouvent en sa personne, l'élu local idéal pour piloter le groupe d'action locale (GAL). Cette nouvelle légitimité lui permet de fédérer les socioprofessionnels autour d'un label "Pays Cathare". Fort de cette réussite, le conseiller général du canton de Mouthoumet peut inscrire son action politique sur le "long terme", car le processus de réappropriation du territoire par les populations rurales "va au-delà d'un mandat électif". La capacité à mobiliser la dimension identitaire du territoire atteste de la nature particulière du leadership politique de ce conseiller général. A partir de là, il développe une conception de l'action politique locale fondée sur "la démocratie participative". Dans cette perspective "le côté identitaire, la croyance en ses valeurs, c'est une élément fondamental du territoire".

Dans la période post-décentralisation où l'intervention communautaire se fait de plus en plus forte, l'analyse comparée de la formation de leadership politique territorial permet de faire avancer la réflexion sur le devenir du pouvoir notabiliaire. La comparaison de la capacité politique des élus locaux a mobilisé le territoire via une conception différenciée de l'action publique et permet de saisir les logiques de recomposition du pouvoir local. Dans la forme notabiliaire traditionnelle, un leader mobilise les ressources sectorielles de l'action publique pour fidéliser une clientèle sur son territoire d'élection. Ainsi, le territoire devient le lieu où se concrétise un échange politique. Il en va différemment quand un élu mobilise les ressources identitaires d'un territoire pour construire son leadership politique. Dans ce cas particulier, le territoire devient le contenu même de l'échange politique comme le montre la stratégie d'action publique globale menée par le conseiller général de Mouthoumet dans le cas du Pays Cathare.

La distinction analytique entre le notable traditionnel et le leadership politique territorial permet d'approfondir la connaissance des nouveaux modes d'action des élus locaux confrontés à la réalité pratique de la décentralisation. Dès lors, il faut s'interroger sur la façon dont les élites locales "transforment les essais" en prenant en compte leur capacité d'adaptation ou de transformation des règles du jeu politique (Bailey, op. cit.). La question de l'accumulation des ressources politiques entraînant la constitution de leaderships territoriaux mérite alors d'être posée dans d'autres configurations. Dans cette perspective, l'analyse de la mobilisation des "ressources culturelles et identitaires" par un leader sur un territoire donné doit être prise en compte de façon plus systématique (Starrat, 1993, pp. 3-4). 


\section{Pôle Sud $N^{\circ} 7$}

L'observation de la formation du leadership politique territorial en compétition pour la maîtrise du projet développement donne un éclairage particulier sur les imbrications entre policy et policies.

De façon plus générale, le développement local pose explicitement la question du rapport au territoire de l'État. Il ne s'agit pour autant d'annoncer à partir de notre étude du projet "Pays Cathare" un retour "des pays d'Etats et en particulier du Languedoc". D'ailleurs, la loi sur l'Aménagement et le développement du territoire (4 février 1995) ouvre la voie à une politique de "contrat de pays" quand ceux-ci présentent "une cohérence géographique, économique, culturelle ou sociale" (Faure, 1995, p. 20). Cependant, il est indéniable que l'État à la française est grignoté par le haut et par le bas, par l'écart entre la mondialisation économique, par la fragmentation, le déclin des grandes institutions et le retour des territoires. Les modifications des cadres institutionnels de l'action publique locale et l'apparition de nouveaux leaderships politiques a ouvert un nouveau débat sur la question de la gouvernance (Le Galès, 1996). L'émergence de cette notion s'inscrit pleinement dans le contexte de la recomposition de l'État et du politique dans les sociétés européennes. Parler de gouvernance, consiste à abandonner le primat de l'État-nation, tout en conservant les idées de conduite, de pilotage, de coordination et d'intégration. S'avancer dans une réflexion sur la reformulation du pouvoir local dans ce cadre analytique entraîne une interrogation sur les rapports entre gouvernance et territoire. Dans cette perspective, l'analyse de la gouvernance territoriale, entendue comme la coordination d'une série d'acteurs politiques, sociaux, économiques, administratifs (notamment sur la forme de réseaux d'action publique locaux) et la capacité d'action collective de cet ensemble d'acteurs, ouvre une piste pour rendre intelligible le changement politique. 


\section{Le territoire imaginaire du Pays Cathare}

\section{Notes}

* Cet article est la version remaniée de "Développement local et gouvernance territoriale : l'exemple du pays cathare", communication présentée au Congrès de l'Association Suisse de Science Politique, Groupe Espace et Politique, Balsthal 8 novembre 1996.

** Cette enquête a été réalisée en collaboration avec Olivier Dedieu et sous la direction d'Emmanuel Négrier dans le cadre du programme de recherche REGE du MZES Manheim sur les réseaux européens de l'Europe 1996.

1. La mobilisation autour de "l'hérésie cathare" se situe au XIIr siècle sur les territoires historiques du Comté de Toulouse et du Languedoc. La croisade et l'annexion par la monarchie française ont mis fin à ce mouvement utopique que certains auteurs ont qualifié de démocratique, égalitaire et émancipateur. Aujourd'hui, un certain nombre de châteaux symbolisent les lieux de la résistance répartis entre les régions Midi-Pyrénées et Languedoc-Roussillon. Le département de l'Aude possède le plus grand nombre de sites cathares: 10 châteaux (L'Histoire, 1994).

2. La mission Racine est la structure qui a planifié dans les années 60 le développement du tourisme sur le littoral méditerranéen.

3. Le Cnec a pour mission l'analyse historique du patrimuine culturel cathare. Le président du Conseil Général de l'Aude déclare en 1982, le jour de l'inauguration du Cnec, qu'"à partir d'aujourd'hui nous sommes tous Cathares" (entretien avec le directeur du Cnec,).

4. On se propose de reprendre ici, dans une acceptation qui nous est propre, l'analyse de la formation des "communautés imaginaires" de Bennedict Anderson (1996). Pour nous, cette forme de communauté peut se développer sur un territoire à partir du moment où un processus de communication à connotation culturelle est effectué sur une population locale. Le travail sur la mémoire historique du Pays Cathare et les symboles identitaires (les châteaux cathares) sont considérés comme les indices de l'institutionnalisation d'un territoire imaginaire.

5. Le directeur du CDPCA se définit comme un méridional, un expert du patrimoine, diplômé des BeauxArts à Toulouse après un travail sur la plastique du catharisme. Il a rédigé une thèse sur l'archéologie de l'architecture des châteaux cathares et la vie au XIII' siècle et a dirigé les fouilles sur les châteaux de Montségur pendant douze ans. De là naît sa vocation pour diffuser un savoir sur le patrimoine cathare. Il travaille avec des élus qui ont un problème de mise en valeur du patrimoine communal.

6. Le directeur du CDPCA s'occupe de constituer le réseau d'experts et de techniciens qui travaille sur la dimension patrimoniale et culturelle du projet (Sarret, 1993, p. 87).

7. Selon le directeur du CDPCA, "la définition géographique du territoire, identifié tant par les populations autochtones que par les visiteurs, s'est imposée sur des bases culturelles : elle est interdépartementale et ne concerne pas seulement des sites majeurs, mais encore tout l'espace, théâtre des événements historiques" (ibid., p. 88).

8. Cette expertise est réalisée par Valérie Patin (1987), Docteur en sociologie et responsable Europe du Comité du tourisme de l'ICOMOS, qui défend la thèse de l'exploitation touristique du patrimoine culturel. Si le patrimoine s'inscrit chaque jour de plus en plus dans une logique économique, il est nécessaire de dépasser l'opposition latente entre les partisans de l'exploitation économique qui risquent de dénaturer les représentations voire détruire le patrimoine et les tenants de la préservation exclusive qui risquent de porter atteinte à l'existence même du patrimoine par l'asphyxie économique et sociale.

9. L'enquête est réalisée en 1989 par le cabinet de consultant "Images Virtuelles - Paris".

10. Il va de soi que dans les populations des territoires concernées, le "Pays Cathare", n'a aucune réalité sociale. Par contre, il en va différemment pour les experts locaux qui portent le projet. Pour ces acteurs, la dimension "faire du développement local autrement" notamment en s'appuyant sur un territoire imaginaire est primordiale (entretien Paul-Marie Durand/Datar).

11. Pour un jeune technicien local qui réalise un stage AFPA, il s'agissait "de pouvoir parler d'un autre tourisme. Ainsi, un monument comme le château de Couiza qui était seulement utilisé médiatiquement 


\section{Pôle Sud $N^{\circ} 7$}

devait faire l'objet de mesure de sauvegarde" (entretien avec l'équipe du Service du Développement Local du conseil général de l'Aude).

12. Paul Marie Durand agissant en tant que représentant de la Datar (via la politique Montagne) constate à ce propos “qu'on retrouve là les professionnels, les élus les services de l'État avec une même convergence. C'est vrai que le département de l'Aude est celui qui a le plus de sites-pôles, mais imaginez un Pays Cathare sans Montségur (Ariège) ou sans Minerve (Hérault)" (CDPCA, 1989, p. 150).

13. On se réfere, ici, à l'opposition qui existe entre les représentations véhiculées par le directeur du CDPCA qui parle du "Pays Cathare" alors que Paul Marie Durand évoque "les Pays Cathares".

14. Ces réunions se déroulent sans aucun formalisme institutionnel, souvent sous la forme "de repas débat" où "l'on refait le monde" (entretien avec le directeur du CDPCA).

15. Entretien avec François Valette, chargé de mission Europe à la délégation régionale LanguedocRoussillon du CNRS, intervenant à titre de consultant sur le programme (15/02/1995).

16. C'est sur cette base qu'un programme Leader Aude, intitulé "Pays Cathare", ayant pour objectif le développement d'une zone d'agro-tourisme dans le département de l'Aude voit le jour. Ce programme vise à encourager une approche intégrée du développement rural et vient compléter les actions qui s'inscrivent dans le cadre communautaire d'appui dans les zones d'objectif 1 et d'objectif $5 b$ (normes européennes). Il s'agit, ici, d'un soutien direct aux initiatives collectives de développement rural prises au niveau local (Paul Alliès, 1994).

17. Il y a eu 217 projets sélectionnés dans la Communauté, 40 en France dont trois en LanguedocRoussillon (Pays Cathare, Montagne-Hérault, SELO en Lozère). Pour une présentation du projet audois par ses représentants, of. Territoires, 1994, pp. 52-54.

18. A ce propos, un des experts locaux qui participe au montage de la version Leader du programme Pays Cathare, nous déclare que les élus locaux sont attentifs aux mises en garde concernant l'utilisation de la subvention européenne. Les logiques de "saupoudrage" du territoire à des fins de clientélisme électoral sont proscrites (entretien François Valette).

19. Il déclare à ce propos : "ces experts savent ce que les gens de Bruxelles ont envie de lire".

20. Il oriente sa vie professionnelle sur l'action de terrain en passant son DEFA (diplôme d'animateur spécialisé en milieu rural).

21. Il garde le mandat de conseiller général et celui maire sans discontinuité en obtenant lors des différentes élections environ $80 \%$ de suffrages.

22. C'est le conseiller général du canton de Mouthoumet qui défend lc projet devant le groupe de la majorité départementale obtenant les 2 millions de francs pour commencer la mise sur agenda de la politique de partenariat nécessaire à la réalisation du projet Leader.

23. Andrieu déclare à propos de la question de l'appropriation par un collectif de son identité "qu'on est parti pour vingt ans et moi je me porte garant du temps".

24. Pour l'anthropologue australien Robert Starrat le renouvellement de l'analyse du leadership passe nécessairement par une prise en compte du cadre culturel de l'action politique des leaders locaux. Dans ce cas particulier, le "leadership substantif" est une forme particulière de leadership qui se fonde sur la mobilisation des identités culturelles. 


\section{Le territoire imaginaire du Pays Cathare}

\section{Références}

Alliès P., "L'impact communautaire sur l'aménagement du territoire", in Finances Locales et Aménagement du Territoire, Paris, Datar \& CLF, 1994, pp. 187-232.

Anderson B., L'imaginaire national. Reflexions sur l'origine et l'essor du nationalisme, Paris, La Découverte, 1996.

Badie B., La fin des territoires, Paris, Fayard, 1995.

Bailey F. G, Les ręgles du jeu politique, Paris, PUF, 1972.

Balme R., Garraud Ph., Hoffmann-Martinot V., Ritaine E., Le territoire pour politique : variations européennes, Paris, L'Harmattan, 1994.

Bayart J.-F., Lillusion identitaire, Paris, Fayard, 1996.

Beniot-Guilbot O., "Les acteurs locaux du développement économique local : y a-t-il un effet localité?", Sociologie du Travaih vol. XXXII, $n^{\circ} 4,1991$, pp. 453-459.

Burns J. Mc G., Leadership, New York, Harper and Row, 1978.

CDPCA, "Le Pays Cathare. Programme pilote de développement interdépartemental (Ariège, Aude, Hérault)", Actes des 5. Rencontres Internationales pour la Protection du Patrimoine Cultureh Avignon 8/9/10 novembre 1989.

Crozier M., Friedberg E., L'acteur et le système. Les contraintes de l'action collective, Paris, Seuil, 1977.

Duran P., Thoenig J.-C., "L'État et la gestion publique territoriale", Revue Fraņ̧aise de Science Politique, vol. 46, $n^{\circ} 4$, août 1996, pp. 580-623.

Faure A., "Les élus locaux à l'épreuve de la décentralisation", Revue Française de Science Politique, vol. 44, n³, 1994, pp. 462-479.

Faure A., "Vers une République régionale", Pouvoirs Locaux, n² 26, Septembre 1995, pp. 21-24.

Faure A., Pollet G., Warin Ph., La construction du sens dans les politiques publiques, Paris, L'Harmattan, 1995.

Fontaine J., Le Bart C., Le métier de l'élu local Paris, L'Harmattan, 1994.

Gaudin J.-P., "Politique urbaines et négociations territoriales. Quelle légitimité pour les réseaux de politiques publiques?", Revue Française de Science Politique, vol. 45, n 1, Février 1995, pp. 31-56.

L'Histoire, $\mathrm{n}^{\circ} 183$ : L'aventure des cathares, Décembre 1994, pp. 38-61.

Lacroix B., L'utopie communautaire, Paris, PUF, 1981.

Le Galès P., "Régulation, gouvernance et territoire", communication à la table ronde $\mathrm{n}^{\circ} \mathbf{2}$ du congrès de l'Association Française de Science Politique, 23-26 avril 1996.

Lorrain D., "De l'administration républicaine au gouvernement urbain", Sociologie du Travail, vol. XXXII, $\mathrm{n}^{\circ} 4$, 1991, pp. 461-484.

Muller P., Les politiques publiques, Paris, PUF, 1990.

Patin Valérie, "L'image du patrimoine et la consommation touristique", communication présentée Les actes des 3. Rencontres Internationales pour la Protection du Patrimoine Culturel, Avignon, novembre 1987, pp. 19-25.

Pôle Sudh $n^{\circ} 4$ : Négociations, mai 1996.

Ritaine E., "Territoires et politique en Europe du Sud", Revue Française de Science Politique, vol. 44, n 1, Février 1994.

Rouban L., Souchon-Zahn M.-F., "L'espace politique en milieu rural. Les maires des communes de moins de 10000 habitants", Les cabiers du CEVIPOF, n 14, Juin 1996.

Sarret Jean-Pierre, "Le pays cathare. Un atout majeur pour le développement", Monuments Historiques, Languedoc-Roussillon, $\mathrm{n}^{\circ}$ 187, 1993.

Smith A., L'Europe au miroir du local Les fonds structurels et les zones rurales en France, en Espagne et au RoyaumeUni Paris, L'Harmattan, 1995.

Sociologie du Travail n 4 : Les Acteurs Du Développement Locah Vol. XXXII, 1991.

Territoires, $n^{\circ} 344:$ Le tourisme se met au vert, janvier 1994, pp. 52-54.

Thœenig J.-C., "Does local politics matter?", débat entre Dominique Lorrain, Jean-Claude Thœnig et Philippe Urfalino, Politix, n 7-8, Octobre-Décembre 1989, pp. 115-123.

Touraine A., Dubet F., Wieviorka M., Le pays contre l'État, Paris, Seuil, 1981. 